\title{
Polyporoid and corticioid Basidiomycetes in pristine forests of the Pechora-Ilych Nature Reserve, Komi Republic, Russia
}

\author{
Ilya A. Viner \\ Moscow State University, Faculty of Biology, Russia. \\ E-mail: viner.ilya@gmail.com
}

\begin{abstract}
New data on non-agaricoid wood-inhabiting basidiomycetes collected from coniferous hosts in Pechora-Ilych Nature Reserve are provided. In total, 182 species were recorded during two field seasons (June-July 2012-2013), of them 64 are reported as new to Komi Republic. Each species in the list below is annotated with host information and specimen collecting data. The paper significantly extends the number of species reported for the region; new records of some rare species, Botryobasidium botryoideum, Chaetoporus mutabilis, Hyphoderma velatum, Physisporinus vitreus, Repetobasidium vile, Tulasnella eichleriana, Tulasnella hyalina, are discussed.
\end{abstract}

Keywords: Polypores, corticioid, biodiversity

\section{INTRODUCTION}

The biodiversity of aphyllophoroid fungi in Komi Republic has been studied since the expedition of E. Parmasto in 1957. He visited three localities in the middle and north zones of taiga forests (Parmasto, 1963, 1967). Later, during 1999-2003, D. A. Kosolapov collected aphyllophoroid fungi in many localities of the middle zone of taiga forests in the Komi Republic and summarized all available data on their biodiversity (Kosolapov, 2008). In comparison to other territories of the Komi Republic, Pechora-Ilych Nature Reserve has been investigated more thoroughly, and numerous publications on fungal biodiversity of this area are available (Hermansson, 1997a-b; Ushakova, 2000a-c; Shiryaev, 2000a-b; Kosolapov, 2008).

The remote eastern part of the reserve is located in the Ural foothills of the upper Pechora river; the landscape is dominated by conifer forests of Abies sibirica Ledeb., Picea obovata Ledeb. and Pinus sibirica Du Tour (Tsyganov et al., 2015). This area is especially interesting for biodiversity studies since there is no forest management and no traces of the crown fires over the last hundreds of years (Aleinikov, unpublished). All these factors are expected to have a positive influence on biodiversity of woodinhabiting fungi due to the positive response of species with limiting dispersal ability and high extinction risk (Nordén et al., 2013) and of species limited to large-sized substrate units (Berglund et al., 2011), which are usually absent in managed and burned forests. As a result, we significantly extended the list of species reported for the Komi Republic. Some findings of rare species are discussed.

\section{MATERIAL AND METHODS}

We collected the data in Pechora-Ilych Nature Reserve, Komi Republic, Russia $\left(62^{\circ} 04^{\prime} 37^{\prime \prime} \mathrm{N}\right.$, $58^{\circ} 57 ' 24$ 'E) in June-July of 2012-2013. Particularly, the study area represents unmanaged taiga forests of the Ural foothills of the upper Pechora river $\left(62^{\circ} 03^{\prime} 07.80^{\prime \prime} \mathrm{N}, 58^{\circ} 57^{\prime} 58.62\right.$ ' $\mathrm{E}$; for habitat description see Tsyganov et al., 2015). In this paper we included findings of polypores and corticioid species, the concept of which generally follows Bernichia and Gorjon (2010), though heterobasidioid taxa such as Basidiodendron spp. were also included in the species list. We surveyed more than 1500 logs of three dominant conifer species: Abies sibirica, Picea obovata and Pinus sibirica. Microscopic identification of species that could not be reliably identified in the field was carried out using $5 \% \mathrm{KOH}$ and Cotton Blue (CB) as recommended by Niemelä (2005). In total, we collected the data on 2265 fruit bodies. The list consists of Latin species name followed by substrate type (one of the three conifer species) and, when available, number of the specimens deposited in personal herbarium, which is available on request from the Zoological Club of Zoological Museum of Moscow University (KUN-ZM-MGU). For nomenclature and accepted names we followed Kotiranta et al. (2009); Myco- 
Bank was also consulted. There was no attempt to include infraspecific taxa since they are not widely used by taxonomists.

\section{RESULTS AND DISCUSSIONS}

In total, 182 species of polyporoid and corticioid Basidiomycetes were collected on dead wood of three conifer species, of them 154 - on Picea obovata, 118 - on Abies sibirica, and 34 - on Pinus sibirica. Almost half of the species grew exclusively on one host, in particular: 53 on Picea obovata, 21 on Abies sibirica and six on Pinus sibirica, while only 22 were observed on all tree host species. Lower diversity of species on pine logs agrees with Renvall (1995) and it is apparently caused by the physical and chemical composition of the pine wood, which is hardlydegradable if compared with Abies sibirica and Picea obovata. We found 65 species, which are new to Komi Republic (Kosolapov, 2008) and also registered three species included in the Red list of Komi Republic: Ganoderma lucidum, Steccherinum collabens and Protomerulius caryae (Taskaev, 2009). G. lucidum and S. collabens show comparatively high abundance in our study plots, indicating that these species are rather common in the study area. This partly contradicts their threat category 3 in the Red book of Komi Republic, because this category implies the sporadic distribution of species (Taskaev, 2009). In turn, P. caryae is included in the threat category 0 , applied for species possibly extinct in Komi Republic (Taskaev, 2009), thus our finding confirms the existence of $P$. caryae in Komi at present (at least on the territory of the Pechora-Ilych Nature Reserve).

We consider the comparatively high fungal diversity of the study area to be caused by several factors: (1) high abundance of large-sized dead wood remnants in old-grow forests; (2) the presence of Abies sibirica as one of the most frequent tree species on the study territory while this phorophyte is absent in the major parts of European Russia; however, it is an obligatory condition for such fungi as Phellinus hartigii and Hymenochaete cruenta (Ryvarden \& Gilbertson, 1994; Bernichia \& Gorjon, 2010); (3) the study area borders on the Siberian part of Russia, favouring the presence of species confined to Asia and almost absent in Europe, e.g. Fomitopsis cajanderi (Ryvarden \& Gilbertson, 1993).

\section{ANNOTATED LIST OF SPECIES}

The species are presented in the alphabetic order. Asterisks $\left(^{*}\right)$ indicate new records for the Komi Republic. The reference consists of a species name in Latin followed by substrate type (log of one of the three conifer species) and, when available, number of the specimen in personal herbarium. Short notes are provided for some records.

Aleurodiscus lividocoeruleus (P. Karst.) P.A. Lemke - Abies sibirica.

Alutaceodontia alutacea (Fr.) J. Erikss. - Abies sibirica, Picea obovata, Pinus sibirica, 406, 408.

Amphinema byssoides (Pers. : Fr.) J. Erikss. Abies sibirica, Picea obovata.

Amylocorticium cebennense (Bourdot) Pouzar Abies sibirica, Picea obovata, 800.

Amylocorticium subincarnatum (Peck) Pouzar Picea obovata.

Amylocystis LAPPONICA (Romell) Singer - Abies sibirica, Picea obovata, 78.

Amylostereum chailleti (Pers. : Fr.) Boidin Abies sibirica, Picea obovata, 776.

*AMYLOXENASMA GRISELlum (Bourdot) Hjortstam \& Ryvarden - Picea obovata.

Antrodia heteromorpha (Fr. : Fr.) Donk - Abies sibirica, Picea obovata, 260, 772, 778.

ANTRODIA SERIALIS (Fr.) Donk - Picea obovata.

Antrodia sinuosa (Fr.) P. Karst. - Abies sibirica, Picea obovata.

Antrodia Xantha (Fr. : Fr.) Ryvarden - Picea obovata, Pinus sibirica, 23.

*Aphanobasidium pseudotsugae (Burt) Boidin \& Gilles - Picea obovata, 731, 796.

*Aphanobasidium subnitens (Bourdot \& Galzin) Jülich - Picea obovata, 764.

Asterodon ferruginosus Pat. - Abies sibirica, Picea obovata.

*Asterostroma cervicolor (Berk. \& M.A. Curtis) Mass. - Abies sibirica, 814.

ATHELIA DECIPIENs (Höhn. \& Litsch.) J. Erikss. Abies sibirica, Picea obovata, Pinus sibirica, 783.

*Athelopsis glaucina (Bourdot \& Galzin) Parmasto - Abies sibirica.

*Athelopsis subinconspicua (Litsch.) Jülich Abies sibirica, Picea obovata, 717, 771 .

*Basidiodendron caesiocinereum (Höhn. \& Litsch.) Luck-Allen - Abies sibirica, Picea obovata, Pinus sibirica, 720. 
*Basidiodendron cinereum (Bres.) Luck-Allen Abies sibirica.

Basidioradulum RADUla (Fr.) Nobles - Abies sibirica, Picea obovata.

BJERKANDERA ADUSTA (Willd : Fr.) P. Karst.- Picea obovata.

*Boidinia furfuracea (Bres.) Stalpers \& Hjortstam - Abies sibirica, 794.

*BoIdinia SUBASPERISPORA (Litsch.) Jülich - Picea obovata, 791.

*Botryobasidium Botryoideum (Overh.) Parmasto - Abies sibirica, Picea obovata, 482, 486, 491, 490, 492, 494. We collected 6 specimens of rare species $B$. botryoideum, which represent the northernmost records of this species. According to the previous records of $B$. botryoideum, it has a discontinuous distribution in Eurasia: the species was registered in the Russian Far East (Langer, 1994), while in Europe it is known from a few localities in Slovakia, Germany, France (Langer, 1994) and Switzerland (Küffer \& Senn-Irlet, 2005). Our findings fill the gap in the known distribution of this species. All studied specimens have subglobose to globose basidiospores with surface varying from slightly asperulate in immature spores to distinctly asperulate in mature ones; hyphae were also slightly to moderately asperulate. We checked the correctness of identification comparing our material with specimens from the Far East (Spirin, unpublished) in CB.

*Botryobasidium CONSPERsum J. Erikss. - Abies sibirica, Picea obovata, 335, 339, 343, 421, 714,716 . No anamorphs were found, which is important for the reliable identification (Bernichia \& Gorjon, 2010).

BOTRYOBASIDIUM INTERTEXTUM (Schwein.) Jülich \& Stalpers - Abies sibirica, Picea obovata, 810.

BOTRYOBASIDIUM MEDIUM J. Erikss. - Abies sibirica, Picea obovata, 761, 770. No anamorphs were found, which is important for the reliable identification (Bernichia \& Gorjon, 2010).

*Botryobasidium obtusisporum J. Erikss. - Abies sibirica, Picea obovata, Pinus sibirica, 340, $713,768$.

BOTRYOBASIDIUM SUBCORONATUM (Höhn. \& Litsch.) Donk - Abies sibirica, Picea obovata, Pinus sibirica, 726.

Botryobasidium VAGUM (Berk. \& M.A. Curtis) D.P. Rogers - Abies sibirica, Picea obovata, Pinus sibirica, 409, 434.
Botryohypochnus isabellinus (Fr.) J. Erikss. Abies sibirica, Picea obovata, 354, 359.

Ceraceomyces borealis (Romell) J. Erikss. \& Ryvarden - Picea obovata, 795.

Ceraceomyces eludens K.H. Larss. - Picea obovata, Pinus sibirica, 727, 730.

CERACEOMYCES SERPENS (Tode: Fr.) Ginns - Picea obovata, 782, 803.

*CERACEOMYCES SUlPhuRINUs (P. Karst.) J. Erikss. \& Ryvarden - Picea obovata.

*Ceraceomyces tessulatus (W.B. Cooke) Jülich - Abies sibirica, Picea obovata, 488, 722.

Ceriporia eXCelsa (S. Lundell) Parmasto - Picea obovata, 787.

${ }^{*}$ CHAETOPORUS MUTABILIS Spirin, Vlasak \& Miettinen nom. prov. - Abies sibirica, Picea obovata, 262, 264, 483, 733, 739, 792. C. mutabilis is treated here as a species with a provisional name since it has not been described yet (Spirin, Vlasak \& Miettinen, unpublished). The species is close to Rigidoporus corticola (Fr.) Pouzar (= Chaetoporus corticola (Fr.) Bondartsev \& Singer) from which could be easily separated by distinctly different shape of spores and a tendency to produce effused-reflexed fruit bodies. Moreover, it seems to prefer dead wood of $A$. sibirica as substrate, while $R$. corticola could be found mostly on Populus tremula L. C. mutabilis has a wide distribution in the Russian Far East; in Europe it is known from a few localities in Nizhny Novgorod and Perm Regions, European part of Russia (Spirin, Vlasak \& Miettinen, unpublished). Our findings in Komi Republic are the northernmost records of this species at present.

Cinereomyces lindBladi (Berk.) Jülich - Abies sibirica, Picea obovata.

Climacocystis boRealis (Fr.) Kotl. \& Pouzar - Picea obovata.

Conferticium ochraceum (Fr. : Fr.) Hallenb. Abies sibirica, Picea obovata, 785.

CONIOPHORA ARIDA (Fr.) P. Karst. - Abies sibirica, Picea obovata.

ConioPHORA OLIVacea (Pers. : Fr.) P. Karst. - Abies sibirica, Picea obovata, Pinus sibirica, 187, 192, 193, 196, 198, 202, 209, 222, 223, 224, 230, 231, 235, 237, 238, 240, 241, 243, 244, 322, 323, 349, 357, 366, 376, 378, 430, 449.

${ }^{*}$ Cristinia helvetica (Pers.) Parmasto - Abies sibirica, Picea obovata, 784, 790.

Crustoderma DRYinum (Berk. \& M.A. Curtis) Parmasto - Abies sibirica, Picea obovata, 391. 
Cylindrobasidium evolvens (Fr. : Fr.) Jülich $-P i-$ cea obovata.

Cystostereum murrayi (Berk. \& M.A. Curtis) Pouzar - Abies sibirica, Picea obovata, 188, 190, 191, 197, 200, 203, 205, 207, 221, 226, 227, 228, 229, 232, 233, 234, 239, $358,361,368,438$.

Dichomitus squalens (P. Karst.) D.A. Reid - Picea obovata, 258.

Dichostereum boreale Pouzar - Abies sibirica, Picea obovata, 312, 313, 314, 315, 316, $317,345,348,351,352,370,396,397$, 401, 411, 417, 437.

Exidia Pithya (Alb. \& Schwein. : Fr.) Fr. - Abies sibirica, Picea obovata.

Fibroporia Gossypium (Speg.) Parmasto - Picea obovata, Pinus sibirica, 2, 779.

Fomitopsis CAJANDERI (P. Karst.) Kotl. \& Pouzar Picea obovata, 477.

Fomitopsis Pinicola (Sw. : Fr.) P. Karst. - Abies sibirica, Picea obovata, Pinus sibirica.

Fomitopsis Rosea (Alb. \& Schwein. : Fr.) P. Karst - Abies sibirica, Picea obovata, 184.

*Galzinia Incrustans (Höhn. \& Litsch.) Parmasto - Pinus sibirica, 721.

Ganoderma lucidum (Curtis) P. Karst. - Abies sibirica.

*Gelatoporia subvermispora (Pilát) Niemelä Abies sibirica, Picea obovata.

Gloeopeniophorella convolvens (P. Karst.) Boidin, Lanq. \& Gilles - Abies sibirica, Picea obovata, 769,789 .

Gloeophyllum abietinum (Bull. : Fr.) P. Karst. Picea obovata, 765.

Gloeophyllum OdoRATum (Wulfen : Fr.) Imazeki Abies sibirica, Picea obovata.

Gloeophyllum SePiarium (Wulfen : Fr.) P. Karst. - Abies sibirica, Picea obovata.

Gloiothele citrina (Pers.) Ginns \& G.W. Freeman - Abies sibirica, Picea obovata, 774.

HETEROBASIDION PARVIPORUM Niemelä \& Korhonen - Abies sibirica, Picea obovata, 77, 189, 201, 253, 325, 454.

Hymenochaete cruenta (Pers.) Donk - Abies sibirica, 195, 208.

Hymenochaete fuliginosa (Pers.) Bres. - Abies sibirica, 328, 741.

Hyphoderma argillaceum (Bres.) Donk - Abies sibirica, Picea obovata.

*Hyphoderma definitum (H.S. Jacks.) Donk - Picea obovata, 797.

*Hyphoderma obTusum J. Erikss. - Picea obovata.
Hyphoderma Setigerum (Fr.) Donk - Abies sibirica, Picea obovata, 355, 362, 379, 431, 450.

*Hyphoderma Velatum K.H. Larss. ad. int.- Abies sibirica, Picea obovata, 719,766 . $H$. velatum is an undescribed species with a provisional name, although it is well known and included into the Finnish checklists of aphyllophoroid fungi (Kotiranta et al., 2009). We failed to find any published records of this species from Russia, however, it has been previously collected in European Russia: (Leningrad and Nizniy Novgorod Regions, and Russian Far East - V. Spirin, pers. comm.). Findings of this species in Finland (Kotiranta et al., 2009) and Russia suggest that $H$. velatum has a widespread distribution with a low abundance in the forest landscapes.

*Hyphodontia alutaria (Burt) J. Erikss. - Abies sibirica, Picea obovata, 382, 425.

Hyphodontia ASPERA (Fr.) J. Erikss. - Abies sibirica, Pinus sibirica, 439.

HyPHODONTIA BREviseTA (P. Karst.) J. Erikss. Abies sibirica, Picea obovata, Pinus sibirica, 310, 311, 344, 386, 389, 403, 405, 422, 435, 436, 442.

HyPHODONTIA CINERACEA (Bourdot \& Galzin) J. Erikss. \& Hjortstam - Picea obovata, Pinus sibirica, 780.

Hyphodontia crustosa (Pers. : Fr.) J. Erikss. Abies sibirica.

*Hyphodontia CURVISPORA J. Erikss. \& Hjortstam - Pinus sibirica, 744.

*Hyphodontia HASTATA (Litsch.) J. Erikss. - Abies sibirica.

*Hyphodontia NESPORI (Bres.) J. Erikss. \& Hjortstam - Abies sibirica.

Hyphodontia Pallidula (Bres.) J. Erikss. - Abies sibirica, Picea obovata, Pinus sibirica, 337, 342, 380, 402, 419, 446.

Hyphodontia subalutacea (P. Karst.) J. Erikss. Abies sibirica, Picea obovata, 416, 429.

*Hypochnicium CREMicolor (Bres.) H. Nilsson \& Hallenb - Picea obovata.

*Hypochnicium cymosum (D.P. Rogers \& H.S. Jacks.) K.H. Larss. \& Hjortstam - Abies sibirica, Picea obovata, 773, 781, 809.

Hypochnicium GeOGenium (Bres.) J. Erikss. - Picea obovata.

*Hypochnicium Polonense (Bres.) A. Strid - Picea obovata, 763.

Hypochnicium Punctulatum (Cooke) J. Erikss. Abies sibirica, Picea obovata. 
*Hypochnicium wakefieldiae (Bres.) J. Erikss. Abies sibirica, Picea obovata, Pinus sibirica, 418, 426.

Ischnoderma BENZOINUM (Wahlenb. : Fr.) P. Karst. - Abies sibirica, Picea obovata, 45, 473.

*LEPTOSPOROMYCES SEPTENTRIONALIS (J. Erikss.) Krieglst. - Abies sibirica, Picea obovata, 732, 736, 767.

Leucogyrophana Romelli (Fr.) Ginns - Abies sibirica.

*Leucogyrophana sororia (Burt) Ginns - Abies sibirica.

Megalocystidium leucoxanthum (Bres.) Jülich Picea obovata, Pinus sibirica, 369.

Meruliopsis taXicola (Pers. : Fr.) Bondartsev Picea obovata, 798, 812.

Mucronella Calva (Alb. \& Schwein.) Fr. - Abies sibirica, Picea obovata.

*Oligoporus Romelli M. Pieri \& Rivoire - Abies sibirica, Picea obovata, 17, 306.

OLIGOPORUS SERICEOMOLLIS (Romell) Bondartseva - Pinus sibirica.

ONNIA LEPORINA (Fr.) H. Jahn - Picea obovata.

Peniophora PITHYA (Pers.) J. Erikss. - Abies sibirica, Picea obovata, 489.

*PeniophoRella Pallida (Bres.) K.H. Larss. - Abies sibirica, Picea obovata, 725.

Peniophorella praetermissa (P. Karst.) K.H. Larss. - Abies sibirica, Picea obovata, Pinus sibirica, 331, 373, 395, 400, 420, 423, 448.

Peniophorella PUBERA (Fr.) P. Karst. - Abies sibirica, Picea obovata, 387, 445, 735, 738, 777.

PERENNIPORIA SUBACIDA (Peck) Donk - Abies sibirica, Picea obovata, Pinus sibirica, 29, 62, 64, 186, 259, 261.

*Phanerochaete calotricha (P. Karst.) J. Erikss. \& Ryvarden - Abies sibirica.

Phanerochaete laevis (Pers. : Fr.) J. Erikss. \& Ryvarden - Abies sibirica, Picea obovata, $71,807$.

Phanerochaete sanguinea (Fr.) Pouzar - Pinus sibirica, 367.

Phanerochaete sordida (P. Karst.) J. Erikss. \& Ryvarden - Abies sibirica, Picea obovata, 718.

*Phanerochaete tuberculata (P. Karst.) Parmasto - Picea obovata, 806.

Phanerochaete velutina (DC. : Fr.) P. Karst. Abies sibirica, Picea obovata, 740, 742.

Phellinus abietis (P. Karst.) H. Jahn - Picea obovata, 76, 254, 452, 453; most probably, its earlier findings (Kosolapov, 2008) were treated as Phellinus chrysoloma (Fr.) Donk sensu lato.
Phellinus ferrugineofuscus (P. Karst.) BourdotAbies sibirica, Picea obovata, 63, 326.

Phellinus hartigi (Allesch. \& Schnabl) Pat. Abies sibirica, Picea obovata, 257, 265, 808.

Phellinus nigrolimitatus (Romell) Bourdot \& Galzin - Abies sibirica, Picea obovata, 34, 346.

Phellinus viticola (Schwein.) Donk - Picea obovata.

Phlebia centrifuga P. Karst. - Abies sibirica, Picea obovata, 356, 364, 374, 377, 383, 384, 398.

*Phlebia FiRMa J. Erikss. \& Hjortstam - Abies sibirica, Picea obovata.

Phlebia gigantea (Fr. : Fr.) Donk - Abies sibirica, Picea obovata, Pinus sibirica, 353, 371, 372, 381, 393, 404.

*Phlebia lilascens (Bourdot) J. Erikss. \& Hjortstam-Abies sibirica, Picea obovata, 329, 375.

Phlebia Livida (Pers. : Fr.) Bres. - Abies sibirica, Picea obovata, 385, 729.

Phlebiella sulphurea (Pers. : Fr.) Ginns \& M.N.L. Lefebvre - Abies sibirica, Picea obovata, Pinus sibirica, 727, 815.

Physisporinus Vitreus (Pers. : Fr.) P. Karst. sensu lato - Abies sibirica, 65, 66, 67, 72, $194,236,350$. There were certain difficulties identifying several polypore specimens belonging to the same species (numbers in the herbarium: 65, 66, 67, 72, 194, 236, $350)$, since all of them were immature and sterile. Nevertheless, the fruit bodies have a distinct appearance (Fig. 1): they are perennial, resupinate, leathery with clearly delimited, white margin; and apricot colored

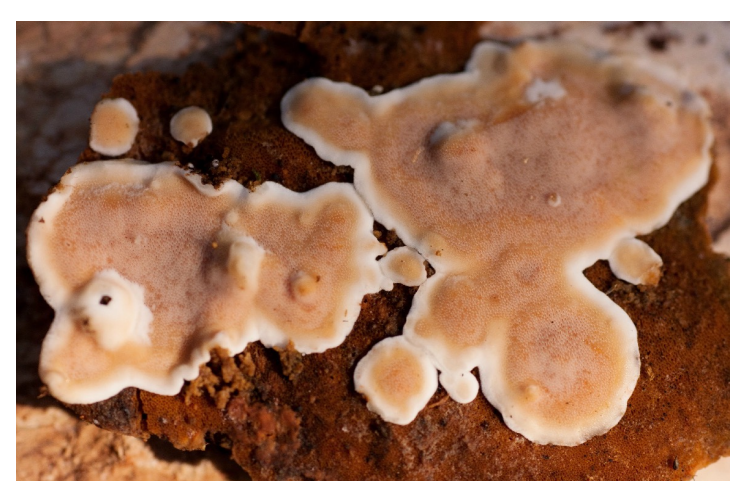

Fig. 1. Physisporinus vitreus sensu lato. The fruit body found on dead wood of Abies sibirica in the Pechora-Ilych Nature Reserve. Photo I. A. Viner. 
pore surface. No color changes were detected in fruit bodies when bruised, though they turned grayish when dry. Hyphal structure is monomitic, clamps and-cystidia are absent. All specimens were collected from moderately decayed logs of Abies sibirica densely covered with mosses. According to the appearance and hyphal structure of fruit bodies we decided to treat it here as $P$. vitreus sensu lato.

Piloderma byssinum (P. Karst.) Jülich - Abies sibirica, Picea obovata.

Postia guttulata (Peck) Jülich - Abies sibirica.

Protomerulius CARyae (Schwein.) Ryvarden Picea obovata.

Pseudomerulius AuReus (Fr.) Jülich - Picea obovata.

Pycnoporellus fulgens (Fr.) Donk - Picea obovata, 3.

*Repetobasidium miRificum J. Erikss. - Abies sibirica, Picea obovata, 485, 493.

*Repetobasidium vile (Bourdot \& Galzin) J. Erikss. - Abies sibirica. R. vile is an uncommon species in Europe, reported from Italy, France, United Kingdom, Sweden, Denmark, Norway, Finland, and Austria (Bernichia \& Gorjon, 2010). Our finding is the first report of $R$. vile for Russia.

Resinicium BICOLOR (Alb. \& Schwein. : Fr.) Parmasto - Abies sibirica, Picea obovata, 318, 319, 320, 321, 327, 334, 360, 363, 390, 392, 412, 427, 433, 441, 443, 451.

*Resinicium furfuraceum (Bres.) Parmasto Abies sibirica, Picea obovata, Pinus sibirica, 332, 336, 341, 365, 399, 424.

Rhodonia Placenta (Fr.) Niemelä, K.H. Larss. \& Schigel - Abies sibirica, Picea obovata, 18, 21, 25, 33.

*Scopuloides Rimosa (W.B. Cooke) Jülich - Abies sibirica, Picea obovata, 728.

*Scytinostroma odoratum (Fr.) Donk - Picea obovata.

*Scytinostromella heterogenea (Bourdot \& Galzin) Parmasto - Picea obovata, 743.

Serpula himantioides (Fr. : Fr.) P. Karst. - Picea obovata.

Sidera LENIS (P. Karst.) Miettinen - Pinus sibirica, 84, 199, 204, 225, 252, 255, 256, 330; microscopically suited well S. lenis sensu stricto, though macroscopically differs in having thicker fruit bodies up to $2 \mathrm{~cm}$ and clearly sinuous pores in old specimens.
Sistotrema BRInKMAnNiI (Bres.) J. Erikss. - Abies sibirica, Picea obovata, 715.

*Sistotrema efibulatum (J. Erikss.) Hjortstam Picea obovata, 801.

*Sistotrema intermedium Hjortstam - Picea obovata.

Sistotrema octosporum (J. Schröt. ex Höhn. \& Litsch.) Hallenb. - Picea obovata.

*Sistotrema Resinicystidium Hallenb. - Abies sibirica, Picea obovata, 793.

SistotremASTRUM NIVEOCREMEUM (Höhn. \& Litsch.) J. Erikss. - Picea obovata.

Sistotremastrum suecicum Litsch. ex J. Erikss. - Picea obovata.

Skeletocutis Amorpha (Fr.) Kotl. \& Pouzar - Abies sibirica, Picea obovata, 19.

Skeletocutis Biguttulata (Romell) Niemelä - Picea obovata.

*Skeletocutis boRealis Niemelä - Picea obovata.

SKELETOCUTIS BREVISPORA Niemelä - Picea obovata.

*Skeletocutis Chrysella Niemelä - Picea obovata.

Skeletocutis KUeHneri A. David - Abies sibirica, Picea obovata, 1, 4, 22, 206, 224, 230.

SkEletocutis OdORA (Sacc.) Ginns - Abies sibirica, Picea obovata.

Skeletocutis Papyracea A. David - Picea obovata, $28,813$.

Steccherinum collabens (Fr.) Vesterh. - Picea obovata, 74, 75, 775 .

STeCCherinum Luteoalbum (P. Karst.) Vesterh. Abies sibirica, Picea obovata, 30, 70, 185.

Steccherinum ochraceum (Pers.) Gray - Picea obovata, 20.

*StecCherinum tenuispinum Spirin, Zmitr. \& Malysheva - Picea obovata.

Stereum SAnguinolentum (Alb. \& Schwein. : Fr.) Fr. - Abies sibirica, Picea obovata, Pinus sibirica.

*ThanatePhORUS FUSisporus (J. Schröt.) P. Roberts \& Hauerslev - Abies sibirica, Picea obovata, $811,786$.

TRECHISPORA CANDIDISSIMA (Schwein.) Bondartsev - Abies sibirica, 26.

TRECHISPORA COHAERENS (Schwein.) Jülich \& Stalpers - Abies sibirica, Picea obovata, 799.

TRECHISPORA FARINACEA (Pers. : Fr.) Liberta - Abies sibirica, Picea obovata, Pinus sibirica, 723, 388.

TRECHISPORA MOlLusCA (Pers. : Fr.) Liberta-Abies sibirica, Picea obovata.

*TRECHISPORA SUBSPHAEROSPORA (Litsch.) Liberta - Picea obovata, 495. 
TRichaptum ABIETinum (Dicks. : Fr.) Ryvarden Abies sibirica, Picea obovata, Pinus sibirica.

TRICHAPTUM FUSCOVIOLACEUM (J.C. Schmidt : Fr.) Kreisel - Abies sibirica, Picea obovata, Pinus sibirica, 456.

TRICHAPTUM LARICINUM (P. Karst.) Ryvarden - Picea obovata (on a standing tree).

*Tubulicrinis accedens (Bourdot \& Galzin) Donk - Pinus sibirica, 724.

TubULICRINIS BOREALIS J. Erikss. - Abies sibirica, Picea obovata, 333, 394, 410.

*Tubulicrinis CAlothrix (Pat.) Donk - Abies sibirica, Picea obovata, 324, 415, 440, 802.

*TUBULICRINIS CHAETOPHORUS (Höhn.) Donk - Abies sibirica, Picea obovata, Pinus sibirica, 734, 805.

Tubulicrinis Glebulosus (Bres.) Donk - Picea obovata, 444.

TubULICRINIS Medius (Bourdot \& Galzin) Oberw. - Abies sibirica, Picea obovata.

*TUBULICRINIS PROPINQUUS (Bourdot \& Galzin) Donk - Picea obovata.

*TUbULICRINIS STRANGULATUS K.H. Larss. \& Hjortstam - Abies sibirica, Picea obovata.

*Tubulicrinis subulatus (Bourdot \& Galzin) Donk - Abies sibirica, Picea obovata, Pinus sibirica, 447.

*Tulasnella Calospora (Boud.) Juel - Abies sibirica, 762.

*Tulasnella eichleriana Bres. - Abies sibirica. Our finding of this heterobasidioid species was expected since it is known from many European countries, and seems to be a widespread in Eurasia (Raitviir, 1967; Roberts, 1994; Roberts \& Hauerslev, 1997); in Russia it has been reported from Sverdlovsk region (Shyryaev et al., 2010).

*Tulasnella hyalina Höhn. \& Litsch. - Picea obovata. T. hyalina is rarely found in Russia. According to the available data, it has previously been collected once in Altai Krai of Russia (Kotkova, 2005).

*Tylospora fibrillosa (Burt) Donk - Picea obovata, $788,804$.

VARARIA InVESTIEns (Schwein.) P. Karst. - Picea obovata.

*VARARIA RACEMosa (Burt) Rog. \& H.S. Jacks. Picea obovata, 484.

Veluticeps abietina (Pers. : Fr.) Hjortstam \& Telleria - Abies sibirica, Picea obovata, 242.

*Xenasma Pruinosum (Pat.) Donk - Abies sibirica, 737.

\section{ACKNOWLEDGEMENTS}

I appreciate my scientific advisers Prof. Irina Sidorova and Tatiana Semenova for constant encouragement. I would like to thank Viacheslav A. Spirin and Otto Miettinen for consultation in identification of some polypores and corticioid species, and the anonymous reviewer for valuable comments on the manuscript. The study was supported by the Russian Foundation for Basic Research, project 13-04-01491 and CIMO International Mobility Centre.

\section{REFERENCES}

Bernicchia, A. \& Gorjón, S. P. 2010. Corticiaceae s. l. Fungi Europaei, vol. 12. Ed. Candusso. 1009 pp.

Berglund, H., Hottola, J., Penttilä, R. \& Siitonen, J. 2011. Linking substrate and habitat requirements of wood-inhabiting fungi to their regional extinction vulnerability. Ecography 34(5): 864-875. http://dx.doi.org/10.1111/j.16000587.2010.06141.x

Hermansson, J. 1997a. Polyporaceae s. lat. and some other fungi in Pechoro-Ilych Zapovednik, Russia. Windahlia (Göteborg) 22: 67-79.

Hermansson, J. 1997b. Members of the family Polyporaceae s. lat. and some other genera of the order Aphyllophorales in Pechoro-Ilychskii reserve. (In Russian). In: Flora i rastitelnost Pechoro-Ilychskogo biosphernogo zapovednika. Yekaterinburg. Pp. 326-365.

Kosolapov, D. A. 2008. Aphyllohoroid fungi of the middle zone of taiga forests in the European NorthEast of Russia. (In Russian). Yekaterinburg, UrO RAS. 229 pp.

Kotiranta, H., Saarenoksa, R. \& Kytövuori, I. 2009. Aphyllophoroid fungi of Finland. A check-list with ecology, distribution, and threat categories. Norrlinia 19: 1-223.

Kotkova, V. M. 2011. To the mycobiota of the Altay Republic. (In Russian, English summary). Novitates systematicae plantarum non vascularium 39: $138-144$.

Küffer \& Senn-Irlet 2005. Diversity and ecology of wood-inhabiting aphyllophoroid basidiomycetes on fallen woody debris in various forest types in Switzerland. Mycological Progress 4(1): 77-86. http: / /dx.doi.org/ 10.1007/s11557-006-0110-z

Langer, G. 1994. Die Gattung Botryobasidium Donk (Corticiaceae, Basidiomycetes). Bibliotheca Mycologica 158: 1-459.

MycoBank. http://www.mycobank.org/ (accessed 29.12.2014).

Niemelä, T. 2005. Käävät, puiden sienet. (In Finnish, English summary). Norrlinia 13: 1-320.

Nordén, J., Penttilä, R., Siitonen, J., Tomppo, E. $\&$ Ovaskainen, O. 2013. Specialist species of wood-inhabiting fungi struggle while general- 
ists thrive in fragmented boreal forests. Journal of Ecology 101(3): 701-712. http://dx.doi. org/10.1111/1365-2745.12085

Parmasto, E. 1963. Data on the fungus flora of the Komi ASSR. (In Russian, English summary). Trudi po botanike 6, Uchenye Zapiski Tartuskogo Gosudarstvennogo Universiteta 136: 103-129.

Parmasto, E. 1967. Polypores in the North of the Soviet Union. Mikologiya i Fitopatologiya 1(4): 280-286.

Raitviir, A. G. 1967. Keys to the Heterobasidiomycetes of the USSR. (In Russian). Leningrad. $113 \mathrm{pp}$.

Renvall, P. 1995. Community structure and dynamics of wood-rotting Basidiomycetes on decomposing conifer trunks in northern Finland. Karstenia 35: $1-51$.

Roberts, P. 1994. Globose and ellipsoid-spored Tulasnella species from Devon and Surrey, with a key to the genus in Europe. Mycological Research 98: 1431-1452. http://dx.doi.org/10.1016/S09537562(09)81075-7

Roberts, P. \& Hauerslev, K. 1997. Tulasnellales Rea. In: Hansen, L. \& Knudsen, H. (eds). Nordic Macromycetes 3. Heterobasidioid, Aphyllophoroid, and Gastromycetoid Basidiomycetes. Copenhagen. Pp. 115-118.

Ryvarden, L. \& Gilbertson R. L. 1993. European Polypores. Part 1. Oslo. Pp. 1-393.

Ryvarden, L. \& Gilbertson R. L. 1994. European Polypores. Part 2. Oslo. Pp. 394-743.

Shiryaev, A. G. 2000a. The clavarioid fungi of the southern part of the Pechoro-Ilychskii Reserve. (In Russian). In: Sostoyanie i dinamika prirodnih kompleksov osobo ohranaemih territoriy Urala. Syktyvkar. Pp. 204-207.
Shiryaev, A. G. 2000b. Relict species of clavarioid fungi from Ural (preliminary report). (In Russian). In: Tezisi dokl. XIV Komi Resp. molodesh. nauch. konf. V 2-h $t$. Syktyvkar. 2: 268.

Shiryaev, A. G., Kotiranta, H., Mukhin, V. A., Stavishenko, I. V. \& Ushakova, N. V. 2010. Aphyllophoroid fungi of Sverdlovsk region, Russia: biodiversity, distribution, ecology and the IUCN threat categories. Yekaterinburg. 303 pp.

Taskaev, A. 2009. Red Data Book of Komi Republic. Syktyvkar. $791 \mathrm{pp}$

Tsyganov, A. N., Komarov, A. A., Mitchell, E. A. D., Shimano, S., Smirnova, O. V., Aleynikov, A. A. \& Mazei, Y. A. 2015. Additive partitioning of testate amoeba species diversity across habitat hierarchy within the pristine southern taiga landscape (Pechora-Ilych Biosphere Reserve, Russia). European Journal of Protistology 51(1): 42-54. http:/ / dx.doi.org/10.1016/j.ejop.2014.11.003

Ushakova, N. V. 2000a. Indicator fungi of old-growth dark conifer forests of Ural. (In Russian). In: Ekologia processov biologicheskogo raslosenia drevesini. Yekaterinburg. Pp. 6-15.

Ushakova, N. V. 2000b. The development of control list of polypore fungi in Ural. (In Russian). In: Mycologia $i$ kriptogammnaya botanica $v$ Rossii: tradicii $i$ sovremennost. Sankt-Petersburg. Pp. 264-266.

Ushakova, N. V. 2000c. The usage of polypores as indicators of nature conditions. (In Russian). In: Sostoyanie $i$ dinamika prirodnih kompleksov osobo ohranaemih territoriy Urala. Syktyvkar. Pp. 186-188. 